Ann. Zootech., I980, 29 (I), 65-86.

\title{
Utilisation de l'énergie des aliments par le veau ruminant. Influence de l'apport de lait, du niveau d'alimentation et de l'âge du veau
}

\author{
M. VERMOREI, J.C. BOUVIER et Y. GEAY*
}

Avec la collaboration technique de Y. Anglaret, J.P. Donnat, Simone Gasnet, R. JAILlER*, C. LEOTy, Marinette Martinadd et R. Souchet

\author{
Labovatoive d'Étude au Métabolisme Énergétique \\ * Laboratoive de la Production de Viande \\ Centre de Recherches de Clermont-Ferrand, I.N.R.A., \\ Theix - Saint-Gènes Champanelle, \\ 63i Iо Beaumont (France)
}

\begin{abstract}
Résumé
Pour analyser l'origine des différences entre les performances des veaux " allaités " et celles des veaux sevrés précocement, on a étudié, par la méthode de calorimétrie indirecte, l'influence d'un apport complémentaire d'aliment d'allaitement distribué sous forme liquide et celle du niveau d'alimentation, sur l'utilisation digestive et énergétique d'un même aliment solide par le veau en croissance, aux âges de 5-ro semaines, 5 mois et 8 mois. Le même aliment solide a été distribué, à 2 niveaux d'alimentation, à des moutons adultes.

Au niveau digestif, il n'y a pas d'intéraction entre l'aliment solide et le lait. Les digestibilités de l'aliment solide ne varient pas avec l'âge des veaux et les teneurs en EM sont comparables à celles obtenues chez les moutons adultes pour le même niveau d'alimentation (tabl. 2). Le rendement d'utilisation de l'EM varie avec l'âge des veaux et la proportion d'énergie apportée par l'aliment d'allaitement (tabl. 4) mais il n'y a pas de différence entre animaux " allaités " et animaux sevrés précocement. Une restriction alimentaire prolongée entraîne une réduction des besoins d'entretien des veaux allaités et améliore leur efficacité alimentaire. Les résultats sont discutés en fonction de la composition des rations.
\end{abstract}

\section{Introduction}

Chez le jeune Ruminant, l'apport de lait naturel ou d'un lait de remplacement entraîne une amélioration des performances : ainsi, LE NEINDRE, PETIT et MULLER (I976) observent que chez le Veau " allaité ", le gain de poids vif et l'efficacité alimentaire augmentent avec la quantité de lait ingérée. Cette amélioration ne 
semble pas due seulement à une augmentation des quantités d'énergie et de protéines ingérées puisqu'on l'obtient également lorsque la même quantité de lait de remplacement est apportée sous forme liquide plutôt que sous forme solide (BI,ACK et TrIDE, I973; GUIIHERMET et al., I977). En effet, le transport du lait naturel ou du lait de remplacement directement dans la caillette par la gouttière osophagienne, permet de fournir à l'organisme des nutriments riches et bien équilibrés. L'association de ces nutriments aux produits terminaux de la digestion microbienne des aliments solides dans le rumen est susceptible de modifier 1'orientation du métabolisme du jeune Ruminant; ainsi, des veaux de race frisonne recevant du lait (veaux. "allaités ") étaient, à poids égal, beaucoup moins gras à l'âge de 9 mois que leurs homologues sevrés précocement (GEAY, I970), mais il faut noter qu'ils ne recevaient pas le même aliment solide.

On a donc essayé d'analyser ce phénomène chez le Veau en croissance par la méthode de calorimétrie indirecte. On a étudié pour cela l'influence d'un apport complémentaire de lait de remplacement (sevrage aux âges de Io semaines ou de 9 mois) et du niveau d'alimentation sur l'utilisation digestive et énergétique d'un même aliment solide, selon l'âge du Veau. La digestibilité et la teneur en énergie métabolisable (EM) de cet aliment solide et le rendement d'utilisation de 1'EM ont été déterminés également chez des moutons adultes en vue de comparer les résultats aux valeurs obtenues chez les veaux aux différents stades de croissance (5 semaines, Io semaines, 5 mois et 8 mois).

\section{Matériel et méthodes}

\section{Animaux}

24 veaux mâles de race Frisonne ont été achetés à l'âge de I7 jours en moyenne, par groupe de 6 , toutes les 2 semaines, afin de pouvoir effectuer les mesures en chambres respiratoires à peu près au même âge chez tous les animaux.

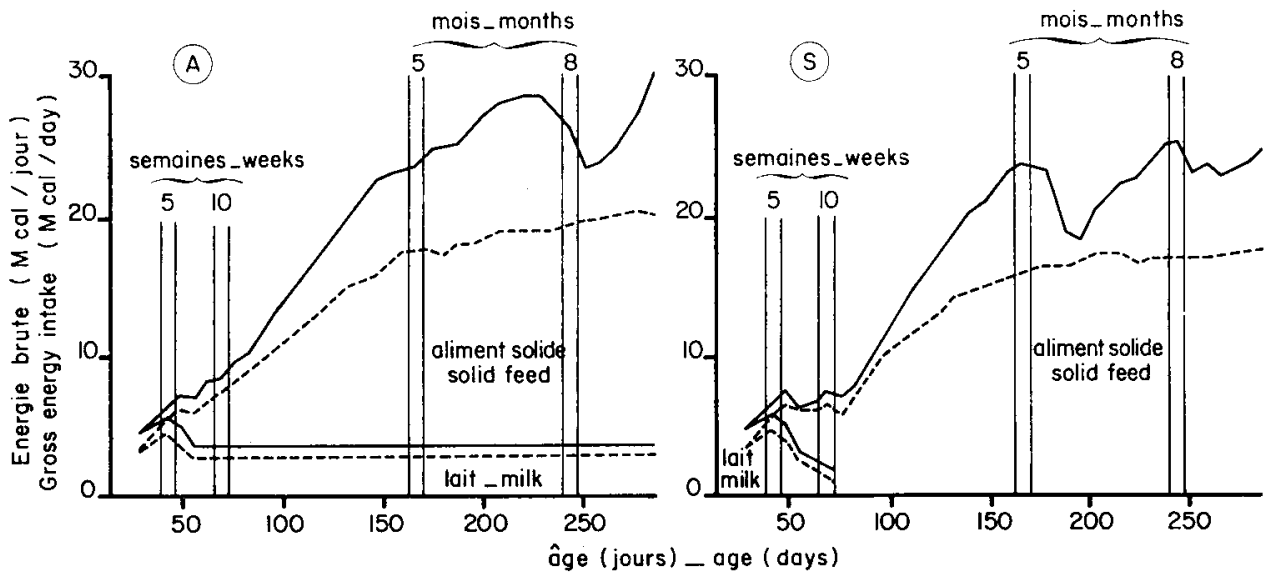

FIG. I. - Variations des quantités moyennes d'énergie brute ingérée par les veaux " allaités " $(A)$ ou sevrés précocement $(S)$, nourris ad libitum (- $(-)$ ou restreints (- - sous forme de lait et d'aliment solide, en fonction de l'àge. Les colonnes représentent les périodes de bilans.

Variations with age in gross energy intake (Mcal/day) of the milk-fed calves $(A)$ or the early weaned calves (S), fed ad libitum (___-) or 75 p. Ioo of ad lib (-..), as milk aczd solid feed. The columns represent the balance periods. 
Six moutons adultes, de race 'Texel, âgés de 2 ans environ et pesant en moyenne $62 \mathrm{~kg}$ ont été utilisés pour les mesures de digestibilité de l'aliment solide et de bilan énergétique.

\section{Mode de conduite et alimentation des animaux}

Les 24 veaux ont été répartis en 4 lots homologues de 5 ou de 7 animaux, en fonction de leur poids à l'arrivée :

- lot $\mathrm{AH}$ (7 veaux) et lot $\mathrm{AB}$ (5 veaux) recevant du lait de remplacement jusqu'à l'âge de 9 mois (système "Veau allaité ") et un aliment solide, soit ad libitum (lot $\mathrm{AH})$, soit en quantités limitées à $75 \mathrm{p}$. Ioo des quantités ingérées par le lot précédent (lot $\mathrm{AB}$ ) (fig. IA);

- lot SH (7 veaux) et lot SB (5 veaux) sevrés à 1'âge de Io semaines (système " sevrage précoce") et recevant le même aliment solide que les lots AH et $\mathrm{AB}$, soit ad libitım (1ot $\mathrm{SH}$ ), soit en quantités limitées à 75 p. Ioo des quantités ingérées par le lot précédent (lot SB) (fig. IB).

Les animaux des lots SH et SB ont reçu respectivement $55 \mathrm{~kg}$ et $43 \mathrm{~kg}$ d'aliment d'allaitement, soit 420 et 330 litres de lait de remplacement. I a répartition au cours des premières semaines est indiquée dans le tableau I. Les animaux des lots $\mathrm{AH}$ et $\mathrm{AB}$ ont reçu les mêmes quantités de lait que ceux des lots $\mathrm{SH}$ et $\mathrm{SB}$ respectivement jusqu'à la $7^{\mathrm{e}}$ semaine incluse et, par la suite, $6 \mathrm{~kg}$ ou $4,5 \mathrm{~kg}$ de lait par jour jusqu'à la fin du $9^{\mathbf{e}}$ mois.

L'aliment d'allaitement comportait I $8 \mathrm{p}$. Ioo de matières grasses et $2 \mathrm{z} \mathrm{p}$. Ioo de matières azotées. Il était distribué en 2 repas égaux par jour, à la dilution de r3o g de poudre par $\mathrm{kg}$ de lait de remplacement. Pour éviter le tri des aliments, tous les animaux recevaient un aliment solide compacté, à I6 p. Ioo de matières azotées par rapport à la matière sèche (MS), constitué de $40 \mathrm{p}$. Ioo de foin de prairie permanente, $44 \mathrm{p}$. Ioo de maïs, I2,5 p. Ioo de tourteau de soja et $3,5 \mathrm{p}$. Ioo de composé minéral et vitaminique. Cet aliment était distribué chaque jour en 2 repas égaux.

TABLEAU I

Variation des quantités de lait distribué ( $\mathrm{kg} /$ /jour) au cours de l'expérience Variations in milk amounts given during the experiment (kg/day)

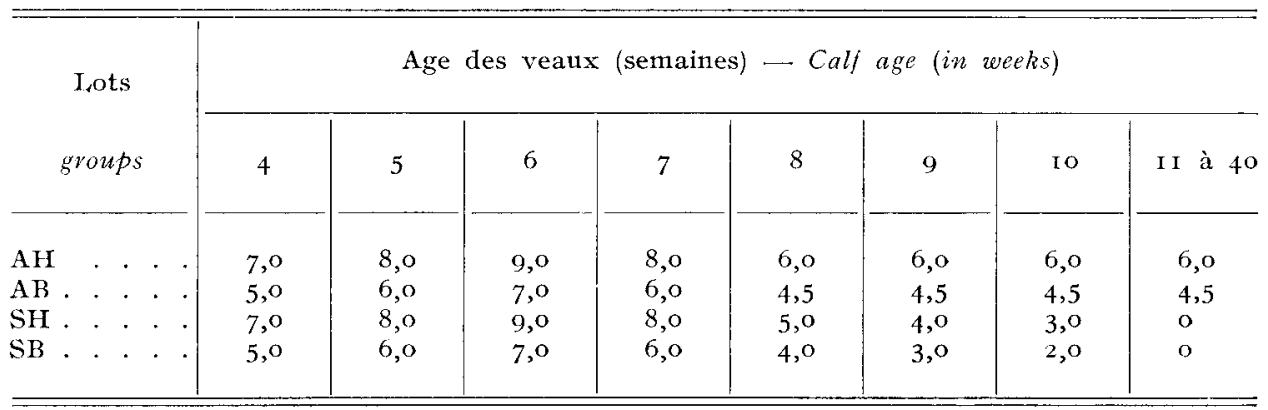


Les 6 moutons ont été répartis en 3 groupes de 2 . Ils ont été adaptés progressivement à l'aliment solide environ 5 semaines avant le début da la période de digestibilité qui précédait les mesures en chambres respiratoires. Ils ont reçu l'aliment solide à 2 niveaux d'alimentation successivement : haut niveau ( $I, 5$ ou $\mathrm{J}, 6 \mathrm{~kg}$ par jour) et bas niveau ( $850 \mathrm{~g}$ par jour).

\section{Mesures}

\section{- Pesée des animaux et quantités d'aliments ingérées}

L,es animaux ont été pesés toutes les 2 semaines, à la même heure. Les quantités de lait et d'aliment solide distribuées et refusées ainsi que les teneurs en MS des aliments et des refus ont été contrôlées chaque jour.

\section{- Digestibilité}

Chez les veaux, au cours de la $6^{\text {e }}$ et de la Io $^{\mathrm{e}}$ semaine d'âge, la digestibilité des aliments a été mesurée pendant des périodes de 6 jours , dans les chambres respiratoires; les veaux étaient équipés de harnais et les fèces étaient collectées dans des sacs en polyéthylène. Aux âges de 5 et $8 \mathrm{~m}$ ois, les fèces ont été récoltées pendant une période de 13 jours consécutifs $(6$ jours dans les chambres respiratoires et 7 jours en cage à digestibilité) directement dans des bacs. Chez les moutons, les mesures de digestibilité ont été effectuées pendant des périodes de 9 jiours en cage à digestibilité.

\section{- Bilan azoté}

Tous lcs animaux étaient équipés de harnais et d'un entonnoir en caoutchouc pour la récolte de l'urine. Cette dernière était pompéo continuellement dans un flacon contenant de l'acide sulfurique. I'urine a été récoltée pendant des périodes de 6 jours cn chantro respiratoire chez le moutons ct les veaux âgés de 5 semaines ou Io semaines et pendant 13 jours chez les veaux âgés de 5 mois ou 8 mois. On a également tenu compte de l'ammoniaque présente dans l'eau condensée sur la batterie froide de la chambre respiratoire.

\section{- Bilan énergétique}

La dépense énergétique des animaux a été déterminée par calorimétrie indirecte à l'aide de 2 chambres respiratoires du type "circuit ouvert" (VERMOREI et al., I973). Les veaux y ont été placés individuellement et les moutons par groupe de 2 . Les échanges respiratoires ont été mesurés pendant 4 jours consécutifs après 3 jours d'adaptation dans les chambres. Les quantités d'énergie fixée ont été déterminées à partir de la production de chaleur (bilan $Q R$ ) et par le bilan carbone-azote (bilan $\mathrm{C}-\mathrm{N}$ ).

\section{- Analyses}

La teneur en énergie brute des aliments frais, des fèces et de l'urine lyopholisées a été déterminée à 1'aide d'un calorimètre adiabatique. Le carbone a été dosé par absorption du $\mathrm{CO}_{2}$ formé dans la bombe calorimétrique. La teneur en azote des aliments frais, des fèces lyophilisées et de l'utine liquide a été déterminée par la méthode macrokjeldah1. 
La signification des résultats relatifs aux performances zootechniques et à l'utilisation digestive des rations a été testée par analyse de variance. Le rendement d'utilisation de 1'EM pour la croissance $(\mathrm{kg})$ a été déterminé par régression de l'énergie fixée en fonction de 1'EM; la signification des rendemients a été testée par analyse de covariance.

\section{Résultats}

I. - Quantités d'aliment ingérćes et performances zootechniques des riaux

Au cours de la période expérimentale, les veaux ont subi 2 épidémies de broncho-pneumonie qui ont affecté très fortement leur consommation et leur croissance pendant plusieurs semaines. Pour la comparaison des traitements, plusieurs animaux particulièrement touchés par cette maladie ont été éliminés et on a conservé 4 ou 5 sujets par lot (tabl. 2). Les performances zootechniques des veaux ont été comparées pendant la période allant du début du sevrage (âge moyen $5^{2}$ jours) à 1'âge de 8 mois ( 247 jours en moyenne) et pendant 2 sous-périodes : des âges de 52 à 160 jours et des âges de I6I à 247 jours. Les quantités d'EM moyennes ingérées par jour au cours de chaque période ont été calculées en multipliant la quantité d'énergie brute ingérée (EI) par la concentration en $\mathrm{E}, \mathrm{M}(q=\mathrm{EM} / \mathrm{EI})$ de chaque ration déterminée pendant les périodes de bilans (cf. $\S 2$ ).

I'apport de $6 \mathrm{~kg}$ de lait par jour n'a entraîné chez les veaux nourris ad libitum qu'une réduction de I $8 \mathrm{p}$. Ioo de la consommation d'aliment solide pendant la première partie de l'expérience et auctne différence significative pendant la deuxième partie et sur l'ensemble de la période expérimentale. En revanche, la quantité d'EM ingérée a été augmentée de 22 p. roo et le gain de poids vif (GMQ) de I9 p. Ioo en moyenne. En conséquence, cet apport de lait n'a provoqué aucune modification significative de l'efficacité globale d'utilisation de 1'EM pour la croissance (GMQ/EM) (tabl. 2).

La restriction alimentaire $(-25$ p. Ioo de 1'EM ingérée en moyenne) a été accompagnée d'une réduction de mème importance du gain de poids vif chez les veaux sevrés précocement et n'a done pas modifié significativemınt l'efficacité alimentaire, tout du moins globalement; l'effet était négatif au cours de la première partie de la période expérimentale mais positif au cours de la seconde. En revanche, chez les veaux " allaités ", le gain de poids vif n'a été réđuit que de I9 p. Ioo et l'efficacité alimentaire a été améliorée de il p. Ioo tout au long de la période expérimentale (tabl. 2).

\section{2. -. Digestibilité et teneur en énergie métabolisable des rations}

Les mesures de digestibilité ont été effectuées sur 8 veaux seulement à 1'âge de 5 semaines ( 4 pour chaque niveau d'aliniantation) en raison du temps limité disponible avant les niesures à 1'âge de ro semaines et sur 20 , I 9 et I 6 veaux aux âges de ro semaines, 5 mois et 8 mois respectivement. 
Variations in calf performance

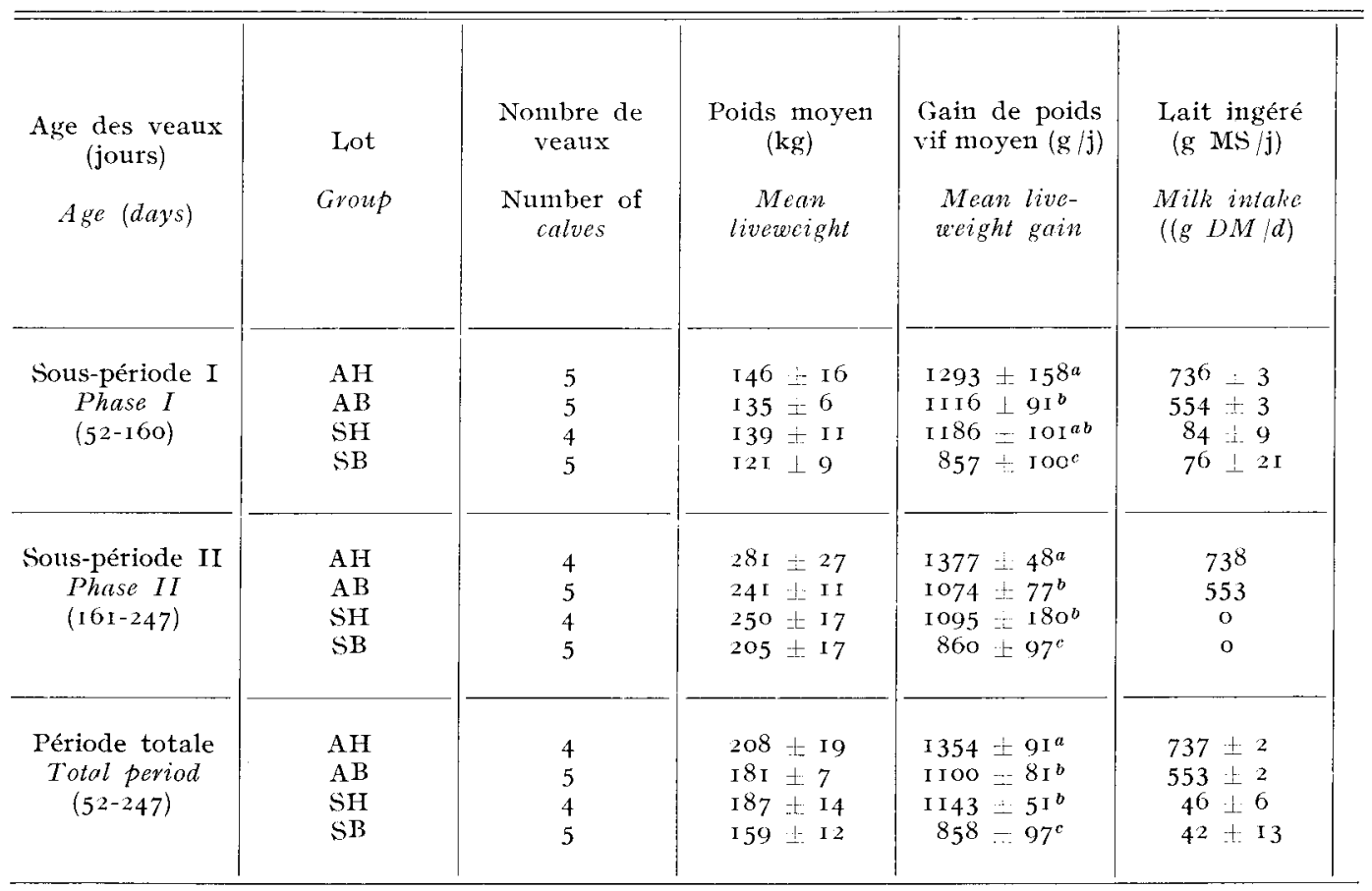

Pour la même colonne et unc période donnée, les valeurs sirmontées de la même lettre ne sont pas significativement différentes $(\mathrm{P}<0,05):{ }^{*}$ GMQ (g)/FM (Mcal); ** GMQ (g)/EM production; EM production = FM totale - EM entretien (Pour chaque lot, le besoin d'entretien correspond aux valeurs déterminées en chambre respiratoire (cf. tabl. 4)).

\section{a) Quantités d'aliment ingérées pendant les périodes de mesure}

A l'âge de 5 semaines, les veaux ont consommé très peu d'aliment solide at l'aliment d'allaitement a fourni en moyenne 86 p. Ioo de l'énergie brute ingérée. Ensuite la corsommation d'aliment solide a augmenté régulièrement jusqu'à l'âge de 6 mois environ chez les veaux nourris ad libitum et est restée à peu près stable jusqu'à la fin de l'expérience (fig. I). L'aliment d'allaitement n'a fourni en moyenne que $30 \mathrm{p}$. Ioo de l'énergie brute à l'âge de Io semaines et $\mathrm{x} 6 \mathrm{p}$. Ioo aux âges de 5 et 8 mois (tabl. 3 ).

Les veaux ont toujours ingéré davantage d'EM par $\mathrm{kg}$ de poids métabolique $\left(\mathrm{P}^{0,75}\right)$ que les moutons. Leur consommation d'aliment solide ( $\mathrm{g}$ de $\mathrm{MS} / \mathrm{kg} \mathrm{P}^{\mathbf{0}, 75}$ ) a été à l'âge de Io semaines voisine de celle des moutons au bas niveau d'alimentation et aux âges de 5 et 8 mois supérieure ou égale à celle des moutons au haut niveau d'alimentation (tabl. 3). Les digestibilités observées chez les veaux devront donc être comparées à celles des moutons pour le haut niveau d'alimentation. 


\section{TABIEAU 2 (suite)}

des veaux (moyennes et écarts-types)

(mean and standard deviation)

\begin{tabular}{|c|c|c|c|c|c|c|}
\hline \multicolumn{3}{|c|}{$\begin{array}{c}\text { Quantités ingérées (par jour) } \\
\text { Intake (por day) }\end{array}$} & \multicolumn{2}{|c|}{$\begin{array}{l}\text { E. Lait /E. ingérée } \\
\text { Milk cnergy \% IE }\end{array}$} & \multicolumn{2}{|c|}{$\begin{array}{l}\text { Efficacité de l'HM } \\
M l: \text { efficioncy }\end{array}$} \\
\hline $\begin{array}{l}\text { Aliment solide } \\
\text { (g MS) } \\
\text { Solid Feed } \\
(g(D M)\end{array}$ & $\begin{array}{l}\text { EM }(\mathrm{kcal}) \\
M E(k c a l)\end{array}$ & $\underset{\mathrm{kcal} / \mathrm{kg} \mathrm{F}^{0,7 i}}{\operatorname{EM}}$ & $\begin{array}{l}\text { E. brute } \\
\text { Ciross. } E\end{array}$ & $\begin{array}{l}E M \\
M E\end{array}$ & $\begin{array}{l}\text { (ilobale* } \\
\text { ()verall* }\end{array}$ & $\begin{array}{l}\text { Partielle** } \\
\text { Partial } * *\end{array}$ \\
\hline $\begin{array}{l}2654+524^{a} \\
2131=245^{b} \\
3228187^{c} \\
2453 \div 210^{a b}\end{array}$ & $\begin{array}{r}10,55 \pm 1,41^{a} \\
8,30 \pm 0,66^{b} \\
9,10 \pm 0,49^{b} \\
6,97 \doteq 0,60^{c}\end{array}$ & $\begin{array}{l}251=16^{a} \\
210 \div 13^{b} \\
225 \div 7^{b} \\
191=12^{c}\end{array}$ & $\begin{array}{r}24,8 \div 3,7 \\
23,3 \pm 2, \mathrm{I} \\
2,9 \pm 0,4 \\
3,5=0,9\end{array}$ & $\begin{array}{r}32,0 \\
30,0 \\
3,7 \\
4,5\end{array}$ & $\begin{array}{l}123 \div 6^{a} \\
135 \pm 7^{b} \\
130 \div 6^{a b} \\
123 \pm 13^{a}\end{array}$ & 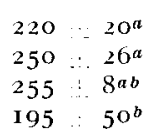 \\
\hline $\begin{array}{l}5213=540^{a} \\
3595 \text { ㄴ } 407^{b} \\
5158+67^{a} \\
3843: 1.35^{b}\end{array}$ & $\begin{array}{l}17,47=1,46^{a} \\
12,25 \pm 1, \mathrm{I}^{b} \\
13,93 \pm \mathrm{I}, 83^{b} \\
10,38 \pm 0,97^{c}\end{array}$ & $\begin{array}{l}255 \pm 5^{a} \\
200 \div 1 \mathrm{I} \\
22 \mathrm{I} \div 26^{c} \\
\mathrm{I} 9 \mathrm{I} \pm 6^{b}\end{array}$ & $\begin{array}{ccc}\mathrm{I} 4,2 & \mathrm{~L} & \mathrm{I}, 3 \\
\mathrm{I} 5,3 & : . & \mathrm{I}, 4 \\
& 0 & \\
& 0 & \end{array}$ & $\begin{array}{c}19,7 \\
20,7 \\
0 \\
0\end{array}$ & $\begin{array}{l}79=8^{a} \\
88=5^{b} \\
78=4^{a} \\
83 \pm 4^{a b}\end{array}$ & 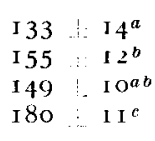 \\
\hline $\begin{array}{l}3841+514^{a} \\
2780+269^{b} \\
4094 \div 22^{a} \\
3077 \div 26^{b}\end{array}$ & $\begin{array}{r}13,76=1,39^{a} \\
10,05 \pm 0,73^{b} \\
1 \mathrm{I}, 27 \pm 0,76^{b} \\
8,50 \pm 0,03^{c}\end{array}$ & $\begin{array}{l}25 \pm \pm 9^{a} \\
204 \pm 1^{b} \\
223 \pm 7^{c} \\
190 \pm 5^{d}\end{array}$ & $\begin{array}{r}18,4 \\
18,9\end{array}$ & $\begin{array}{r}24,9 \\
25,0 \\
1,7 \\
2, \mathrm{I}\end{array}$ & $\begin{array}{l}99 \div 5^{a} \\
110 \perp 3^{b} \\
102 \div 4^{a} \\
101 \div 6^{a}\end{array}$ & $\begin{array}{lll}\mathrm{I} 7 \mathrm{I} & \mathrm{I} 2^{a} \\
\mathrm{I} 96 & \mathrm{I} \mathrm{I}^{b} \\
\mathrm{I} 94 & \mathrm{I} \mathrm{O}^{b} \\
228 & \mathrm{I} 6^{c}\end{array}$ \\
\hline
\end{tabular}

In the same column, means with same supcrscripts are not significantly different $(P \ll 0.05)$ : * live weighr gain (g)/ME (Mcal); ** live a'eight gain/ME for production;

$M E$ for production $=M E-M E$ for maintenance

(In each case, the maintenance requirement amounts to the value determined in this expcriment (table 4 )).

b) Digestibilité et teneur en EM

\section{- Veaux}

Les digestibilités de l'énergie et de l'azote des rations distribuées aux veaux "allaités" ont toujours été plus élevées que celles des veaux sevrés précocement. Flles dépendent directement du pourcentage (L) d'énergie brute apportée par l'aliment d'allaitement dans la ration totale. Les CUI) de l'énergie et de l'azote diminuent aussi significativement $(P \leqslant 0,0$ I) de 2,7 et 3,0 points respectivement lorsque le niveau d'ingestion (NI) augmente d'une unité (relations I et 2). Le niveau d'ingestion unitaire a été défini comme la quantité d'aliment solide nécessaire pour satisfaire le besoin d'entretien des animaux (40 $\mathrm{g}$ de MS par $\mathrm{kg}$ $\left.\mathrm{P}^{0,75}\right)$. En revanche, les digestibilités de 1'énergie $\left(\mathrm{CUD}_{\mathrm{N}}\right)$ et de 1'azote (CUD $)$ ne varient pas significativement avec 1'âge des veaux :

$$
\begin{array}{llll}
\operatorname{CUD}_{\mathrm{E}}=0,2 \mathrm{r} 23 & \mathrm{~L}-2,68 \mathrm{NI}+75,2 \mathrm{I} & \mathrm{R}^{2}=0,902 & \mathrm{~S} x y=2,00 \\
\operatorname{CUD}_{\mathrm{N}}=0,1946 \mathrm{~L}-2,98 \mathrm{NI}+76,84 & \mathrm{R}^{2}=0,888 & \mathrm{~S} x y=\mathrm{I}, 99
\end{array}
$$

(Sxy, écart type résiduel de la régression) 


\section{TABLEAU 3}

Lariations des digestibilités et de la teneur en énergie métabolisable lariations in energy and nitrogcn digestibility and metabolizability of the rations

\begin{tabular}{|c|c|c|c|c|c|c|}
\hline \multirow{2}{*}{$\begin{array}{c}\text { Animaux (A nimals) } \\
\text { Age (jours) } \\
\text { Age (days) }\end{array}$} & \multirow{2}{*}{$\begin{array}{l}\text { Lot } \\
\text { Group }\end{array}$} & \multirow{2}{*}{$\begin{array}{l}\text { Nombre } \\
\text { Number }\end{array}$} & \multirow{2}{*}{$\begin{array}{l}\text { Poids moyen } \\
\text { Mean } \\
\text { weight } \\
\text { (kg) }\end{array}$} & \multicolumn{2}{|c|}{$\begin{array}{l}\text { Ingere }- \text { Intake } \\
\quad(/ \mathrm{kg} \mathrm{P} 0,75)\end{array}$} & \multirow{2}{*}{$\begin{array}{l}\text { E. 1ait \% EI } \\
\text { Milk \%IE }\end{array}$} \\
\hline & & & & $\begin{array}{l}\text { Alim. solide } \\
\text { (g MS/j) } \\
\text { Solid /"ed }\end{array}$ & $\begin{array}{l}\mathrm{EM}(\mathrm{kcal} / \mathrm{j}) \\
M E(\text { kcal } / d)\end{array}$ & \\
\hline Veaux (5 semaines) & $\mathrm{AH}$ & 4 & $73 \pm 8$ & $6,2 \perp 2,0^{a}$ & $223 \pm 19^{a}$ & 90,6 \\
\hline $\begin{array}{l}\text { Calves (5 weeks) } \\
(39 \pm 3)\end{array}$ & $\mathrm{AB}$ & 4 & $66 \div \mathrm{I}$ & II $, 0 \pm 4,2^{a}$ & $203=13^{n}$ & $8 \mathrm{I}, 9$ \\
\hline $\begin{array}{l}\text { Veaux (Io semaines) } \\
\text { Calves (Io weeks) } \\
\quad(70 \quad \therefore 5)\end{array}$ & $\begin{array}{l}\mathrm{AH} . \\
\mathrm{AB} \\
\mathrm{SH} \\
\mathrm{SB}\end{array}$ & $\begin{array}{l}5 \\
5 \\
5 \\
5\end{array}$ & $\begin{array}{l}97 \pm 9 \\
88=3 \\
89=7 \\
85=11\end{array}$ & $\begin{array}{l}35, \mathbf{I}: 5,7^{b} \\
37,3 \div 2,3^{b c} \\
4^{8,3} \mathbf{5}, 9^{d} \\
4^{0,5} \mathbf{3}, 6^{c}\end{array}$ & $\begin{array}{l}216 \pm 16^{a} \\
190 \pm 13^{b} \\
\mathrm{I} 86 \pm 17^{b} \\
159 \pm 20^{c}\end{array}$ & $\begin{array}{l}42,6 \\
37,5 \\
23,6 \\
20,4\end{array}$ \\
\hline $\begin{array}{l}\text { Veaux ( } 5 \text { mois) } \\
\text { Calves ( } 5 \text { months) } \\
\text { (I67 } \because 8)\end{array}$ & $\begin{array}{l}\mathrm{AH} . \\
\mathrm{AB} . \\
\mathrm{SH} . \\
\mathrm{SB} .\end{array}$ & $\begin{array}{l}5 \\
5 \\
5 \\
4\end{array}$ & $\begin{array}{l}215=26 \\
201=15 \\
204=20 \\
17821\end{array}$ & $\begin{array}{l}7 \mathrm{I}, 2 \div 10, \mathrm{I}^{g} \\
63, \mathrm{I}-4,7^{e} \\
94,7 \div 6,3^{h} \\
77,7 \perp 2,2^{g}\end{array}$ & $\begin{array}{l}259 \pm 29^{d l} \\
225 \pm 14^{a} \\
254 \pm 17^{d} \\
209 \pm 6^{a}\end{array}$ & $\begin{array}{l}\text { I } 8,0 \\
16,5 \\
0 \\
0\end{array}$ \\
\hline $\begin{array}{l}\text { Veaux (8 mois) } \\
\text { Calves }(8 \text { months }) \\
\quad\left(24^{2}+\text { II }\right)\end{array}$ & $\begin{array}{l}\mathrm{AH} . \\
\mathrm{AB} . \\
\mathrm{SH} . \\
\mathrm{SB}\end{array}$ & $\begin{array}{l}4 \\
4 \\
4 \\
4\end{array}$ & $\begin{array}{l}33 \mathbf{I}: 23 \\
285 \div 23 \\
286 \div 22 \\
243 \div 20\end{array}$ & $\begin{array}{l}63,5 \ldots 8,1^{e} \\
56,3 \perp 3,5^{f} \\
72,7 \div 7,2^{g} \\
64,9 \vdots 1,0^{e}\end{array}$ & $\begin{array}{l}2 \mathrm{I} 2 \pm 33^{a} \\
\mathrm{I} 89 \pm 10^{b} \\
20 \mathrm{I}=20^{a b} \\
\mathrm{I} 73 \pm 3^{c}\end{array}$ & $\begin{array}{l}\text { I } 5,3 \\
\text { I } 4,0 \\
0 \\
0\end{array}$ \\
\hline $\begin{array}{l}\text { Moutons ( } 2 \text { ans) } \\
\text { Sheep (2 years). }\end{array}$ & $\begin{array}{l}\text { Haut } \\
\text { High } \\
\text { Bas. } \\
\text { Low. }\end{array}$ & 6 & $\begin{array}{l}64 \pm 2 \\
61 \pm 2\end{array}$ & $\begin{array}{l}6 \mathrm{I}, 3: \mathrm{I}, \mathbf{5}^{e} \\
35,4 \perp \mathrm{I}, \mathrm{o}^{b}\end{array}$ & $\begin{array}{l}167 \pm 4^{c} \\
102 \pm 3^{e}\end{array}$ & o \\
\hline
\end{tabular}

Pour une même colonne, les valeurs surmontées de la mêne lettre ne sont pas significativement différentes $(P<0,05)$

Pour $\mathrm{I}_{\mathrm{L}}=$ roo et pour le niveau d'ingestion moyen $(\mathrm{NI}=\mathrm{I}, 65)$ on obtient pour l'aliment d'allaitement des valeurs de 92,0 et $9 I, 4$ pour les CUD de l'énergie et de 1'azote respectivement, non significativement différentes de celles (92,8 et $92,9)$ obtenues pour le même aliment d'allaitement chez des veaux préruminants (VERMOREL, BOUVIER et GEAY, I976).

Les pertes d'énergie sous forme de méthane augmentent avec l'âge des veaux. Négligeables à l'âge de 6 semaines, elles représentent en moyenne 2,6-3,8 et 4,9 p. Ioo de l'énergie ingérée aux âges de ro semaines, 5 mois et 8 mois (tabl. 3 ). Elles sont plus faibles chez les veaux "allaités " que chez les veaux sevrés précocement mais, exprimées en pourcentage de l'énergie apportée par l'aliment solide, 
TABLEAU 3 (suite)

des rations chez les veaux et les moutons (moyennes et écarts-types)

in growing calves and adult sheep (mean and standard deviation)

\begin{tabular}{|c|c|c|c|c|c|}
\hline CUD énergie & CUD azote & E. $\mathrm{CH}_{4} \% \mathrm{EI}$ & E. urine $\% \mathrm{EI}$ & EMI \% IiI & $\mathrm{EM} \% \mathrm{ED}$ \\
\hline E. digest. & N. digest. & $\mathrm{CH}_{4} \mathrm{E} \% \mathrm{IE}$ & Urine $E \% I E$ & $M E \% \quad I E$ & ME $\%$ IIE \\
\hline 90,6 上 I, $8^{a}$ & $88,9: \mathrm{I}, 7^{a}$ & 一 & $1,96-0,26^{a}$ & $88,6 \div 1,9^{a}$ & $97,8 \pm 0,3^{a}$ \\
\hline $88,0 \div 1,5^{a}$ & $87,6-\mathrm{I}, \mathrm{I}^{a}$ & - & $2,35=0,14^{n c}$ & $85,6 \pm 1,4^{b}$ & $97,3 \pm 0,2^{a}$ \\
\hline $80,8 \therefore 3,3^{b}$ & $8 \mathrm{I}, 7 \quad 2,8^{b}$ & $2,33 \div 0,5^{8 n}$ & $3,03 \div 0,32^{b}$ & $75,53,3^{c}$ & $93,4 \pm c, 8^{\circ}$ \\
\hline $78,9 \div 2,6 b c$ & $8 \mathrm{C}, 4=1,8^{\circ}$ & $2,47 \pm 0,47^{a}$ & $3,39+0,23^{\circ}$ & $73,1-2,4^{c d}$ & $92,6 \doteq 0,8^{b}$ \\
\hline $73, \mathrm{I} \pm \mathrm{I}, \mathbf{1}^{a}$ & $74, \mathrm{I} \perp \quad \mathrm{I}, 9^{c}$ & $2,74+0,5 \mathrm{I} a$ & $2,52 \div 0,4^{c}$ & $68,0=1,2^{e}$ & $93,0 \div \mathrm{r}, 2^{b}$ \\
\hline $7^{6,6}$. $2,8^{c}$ & $76,8 \quad !-2,8^{a}$ & $2,75 \div 0,35^{n}$ & $2,5^{8} \perp 0,10^{\circ}$ & $7 \mathrm{I}, 3 \pm 3,2^{d}$ & $93,0 \pm 0,8^{b}$ \\
\hline $72,5+2,3^{a}$ & $75,2: 1,3^{c d}$ & $3,06+0,49^{a b}$ & $3,31 \pm 0,44^{b}$ & $66,2 \pm 1,9^{f g}$ & $9 \mathrm{I}, 2 \perp \mathrm{I}, \mathrm{O}^{\circ}$ \\
\hline $75,3 \perp 3,0^{c d e}$ & 77,7 i $1,8^{d}$ & $3,5^{6}=0,5^{b c}$ & $3,35 \perp 0,23^{b}$ & $68,4 \pm 3,0^{f}$ & $90,8 \pm 0,7^{c}$ \\
\hline $69,5 \div 1,6^{f}$ & $7^{1}, 0 \quad 0,9^{e}$ & $4, \mathrm{OI}=0,32^{\mathrm{edl}}$ & $3,05=0,09^{b i}$ & $61,6 \div 1,4^{n}$ & $89,0 \div 0,4^{d}$ \\
\hline $70,2-1,2^{f}$ & $72,5: \mathrm{I}, 2^{f}$ & $4,65 ! 0,7^{8 d}$ & $3,7 \mathrm{I} \quad 0,0 \mathrm{I}^{d}$ & $61,8+0,9^{h}$ & $88, \mathrm{I} \perp \mathrm{I}, \mathrm{O}^{d}$ \\
\hline $7 \geq, 7 \quad \therefore \quad \mathrm{I}, \mathrm{I}^{d f}$ & $73,8 \ldots \ldots \quad 1,9^{c f}$ & 3,85 : $0,28 b c d$ & $4,13=0,59^{e}$ & $65,0 \div 1,9^{y}$ & $89,0=\mathrm{I}, 2^{d}$ \\
\hline $74, \mathrm{I} \perp \mathrm{I}, 5^{d e}$ & $75,4: 1,3^{c d}$ & $4,35 \perp 0,60^{r d l}$ & $3,36=0,08 b d$ & $66,4 \perp I, 5^{f g}$ & 89,6 -i $0,8^{a}$ \\
\hline $7 \mathrm{I}, 6 \quad \mathrm{i}, 7^{d f}$ & $71,0 \perp 0,6^{e}$ & 4,67 i $0,66^{\prime}$ & $3,4^{\circ} \perp 0,12^{b d}$ & $63,5 \pm \mathrm{I}, 3^{g h}$ & $88,8 \perp 0,9^{d}$ \\
\hline 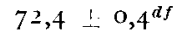 & $7 \mathrm{c}, 4 \quad 1 . .1,4^{e}$ & 6,61 i $0,88 e$ & $4,0_{3}=0,30^{d}$ & $61,2=0,6 h$ & $8_{5,3} \pm 0,9^{e}$ \\
\hline $72,2=\mathrm{I}, \mathrm{O}^{d f}$ & $72,2: 0,6^{c e f}$ & $6,02 \ldots 1,05^{e}$ & $3,62-0,26^{0 d}$ & $62,5=1,2^{h}$ & $86,6 ! 1, \mathrm{r}^{f}$ \\
\hline $77,6 \pm 0,5^{g}$ & 77,8 : $0,7^{a}$ & $7,7^{2} \pm 0,37^{f}$ & $4,08=0,29^{e}$ & $66,2 \pm 0,9^{g}$ & $84,9 \pm 0,7^{g}$ \\
\hline
\end{tabular}

(In the same column, means reith the same superscript are not significantly different $(P<0.05))$.

elles ne sont pas significativement différentes. Elles dépendent donc à la fois de l'âge des veaux ( $T$, en jours) et du pourcentage ( $L$ ) d'énergie brute apportée par le lait dans la ration totale (relation 3 ); en revanche, elles ne diminuent pas significativement lorsque le niveau d'alimentation augmente, malgré la tendance visible aux âges de 5 et 8 mois.

E. $\mathrm{CH}_{4} \% \mathrm{EI}=0,00745 \mathrm{~T}-0,0403 \mathrm{~L}+3, \mathrm{I} 95 \quad \mathrm{R}^{2}=0,659 \quad \mathrm{~S} x y=0,75 \quad$ (3)

Les pertes d'énergie urinaire ne sont pas significativement différentes entre les veaux "allaités" et les veaux sevrés précocement. Elle augmentent avec l'âge des veaux, passant de 2,2 p. Ioo de l'énergie ingérée à l'âge de 5 semaines à 3,7 p. Ioo à l'âge de 8 mois. 
Comme le CUI) de l'énergie, la concentration en EM ( $q=$ roo EM/EI) des rations est plus élevée chez les veaux " allaités " que chez les veaux sevrés précocement. Elle dépend du pourcentage (L) d'énergie brute apportée par l'aliment d'allaitement, du niveau d'ingestion (NI) et, comme le méthane, de l'âge des veaux ( $\mathrm{T}$, en jours) (relation 4 ).

$q=-0,0 \mathrm{Ir} 25 \mathrm{~T}-\mathrm{I}, 92 \mathrm{NI}+0,263 \mathrm{~L}+67,83 \quad \mathrm{R}^{2}=0,940 \quad \mathrm{~S} x y=2,04 \quad$ (4)

En conséquence, le rapport entre l'énergie métabolisable et l'énergie digestible (EM/ED) diminue progressivement, passant de 0,97 à l'âge de 5 semaines à 0,87 à l'âge de 8 mois chez les veaux sevrés précocement (tab1. 3).

- Moutons :

Les CUD de l'énergie et de l'azote de l'aliment solide distribué aux moutons au haut niveau d'alimentation ne sont pas significativement différents de ceux obtenus chez les veaux sevrés précocement, aux âges de 5 et 8 mois, et des valeurs données par les relations I et 2 pour le même niveau d'ingestion. En revanche, les CUD de l'énergie et de l'azote diminuent de 5,5 points lorsque le niveau d'alimentation passe de $I, 08$ à $I, 76(P \leqslant 0,00 I)$ (tabl. 3$)$. Cette observation est confirmée par les résultats d'une vérification effectuée dans les mêmes conditions avec 6 autres moutons ingérant les mêmes quantités d'un aliment très voisin : le CUD de la MS diminue de 3,9 $\pm \mathrm{I}, 4$ points entre les 2 niveaux d'alimentation.

La perte d'énergie sous forme de méthane diminue significativement lorsque le niveau d'alimentation augmente $(P \leqslant 0,025)$ et elle est comparable chez les moutons adultes et chez les veaux âgés de 8 mois, pour le même niveau d'alimentation. L'énergie urinaire n'est pas significativement plus faible au haut niveau d'alimentation et elle est comparable chez les moutons adultes et chez les veaux sevrés précocement âgés de 8 mois, pour le même niveau d'alimentation (tabl. 3 ).

Ces faibles diminutions des pertes d'énergie urinaire et sous forme de méthane ne compensent que partiellement la forte réduction du CUD de l'énergie due à l'augmentation du niveau d'alimentation. La concentration en EM $(q)$ est inférieure de 3,7 points au haut niveau d'alimentation $(\mathrm{P} \leqslant 0, \mathrm{OOI})$ par rapport au bas niveau (tabl. 3). Par ailleurs, elle n'est pas significativement différente des valeurs calculées à l'aide de la relation (4) pour l'aliment solide, au niveau d'ingestion de $x, 76$, chez les veaux $(63,7-62,6$ et $6 \mathrm{r}, 7$ aux âges de ro semaines, 5 mois et 8 mois respectivement).

\section{3. - Utilisation de l'énergie métabolisoble par les veaux}

Sur un total de 63 bilans énergétiques, 6 ont dû être éliminés : 4 en raison de la dérive d'un débitmètre et 2 en raison de fortes variations des échanges respiratoires des veaux au cours des 4 jours de mesure. On dispose en moyenne de 4 bilans énergétiques par traitement et pour chaque stade de croissance.

La concordance entre les résultats des bilans $Q R$ et $\mathrm{C}-\mathrm{N}$ est satisfaisante puisque la différence d'énergie fixée est en général inférieure ou égale à $\mathrm{I}$ p. Ioo de l'énergie ingérée pour l'ensemble des mesures sur veaux et moutons. On n'a cependant utilisé que les données du bilan $Q R$ qui conduisent à des résultats plus homogènes.

\section{Rendement d'utilisation de l'EM pour la croissance}

A 1'âge de 5 semaines, 1'aliment d'allaitement fournit en moyenne $86 \mathrm{p}$. roo de l'énergie brute ingérée. La régression de l'énergie fixée sur l'EM est la même 
pour les veaux nourris ad libitum et pour les veaux restreints depuis une semaine seulement (fig. 2). Le rendement $\mathrm{kg}(0,638$ 土 $0, \mathrm{IOO})$ est légèrement inférieur mais non significativement différent de ceux obtenus chez des veaux préruminants par Van ES (I970) : 0,687; VERMOREL et al., ( 974) : 0,694; VERMOREL, BOUVIER et GEAY (I976) : 0,667 .

A l'âge de ro semaines, l'aliment d'allaitement fournit 40 et $22 \mathrm{p}$. Ioo de l'énergie brute ingérée, respectivement par les veaux " allaités" et sevrés précocement. L'analyse de covariance permet de distinguer 2 populations différentes : les veaux recevant l'aliment solide ad libitum d'une part et les veaux recevant 1'aliment solide en quantité limitée (veaux restreints) d'autre part (fig. 3). Si les moyennes ajustées sont significativement différentes $(F=16,7)$, les pentes ne le sont pas $(F=0, I 7)$. La pente commune correspond à un rendement $(\mathrm{kg})$ de $0,507 \pm 0,033$, très inférieur à celui obtenu à 1'âge de 5 semaines. Cependant, ce rendement est associé à un besoin d'ertretien plus faible qu'aux âges de 5 semaines, 5 mois et 8 mois, mais non significativement différent. Ce phénomène peut résulter de la variabilité ces données expérimentales. Si le besoin d'entretien était le même qu'aux autres âges, le rendement correspondant serait de 0,57 (tab1. 4).

A l'âge de 5 mois, l'aliment d'allaitement ne fournit plus que i 6 et o p. Ioo đe l'énergie ingérée respectivement par les veaux " allaités " et sevrés précccement. On obtient 2 populations différentes : l'une correspondant aux lots $\mathrm{AH}$, SH et SB, l'autre au lot AB (fig. 4).Les moyennes ajustées sont significativement différentes $(\mathrm{F}=32)$ mais pas les pentes $(\mathrm{F}=0,09)$. La pente commune correspond à un rendement $(\mathrm{kg})$ de $0,491 \pm 0,036$.

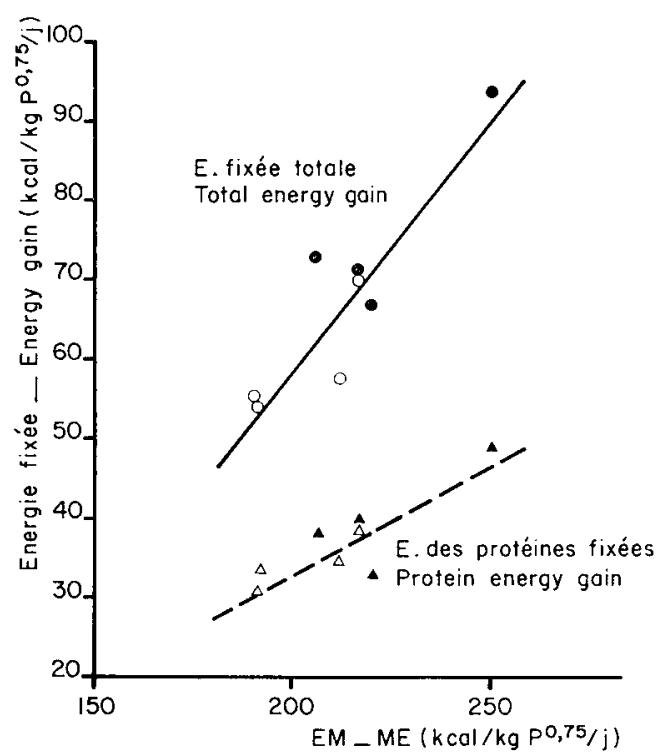

Iirg. 2. - Variations des quantités d'énergie fixée totale et sous forme de protéines par les veaux nourris ad libitum $(\bullet \mathbf{A})$ ou restreints $(O \Delta)$ à l'âge de 5 semaines, en fonction de la quantité d'EM ingérée.

Variations with $M E$ intake, in total cnergy gain and protein cnergy gain of the 5 week-old calves fed ad libitum ( $\mathbf{A}$ ) or $75 \mathrm{p}$. Ioo of ad libitum $(\mathrm{O} \Delta)$.

Fnergie fixée totale (Total energy gain) : $\mathrm{Y}=(0,638 \pm 0, \mathrm{r}$ oo $) \mathrm{EM}-68,9( \pm 5,0) r=0,932$. Energie des protéines fixées (Protein encrgy gain) :

$$
\mathrm{Y}=(0,2574 \pm 0,064) \mathrm{EM}-\mathrm{I} 7,9( \pm 3,2) \quad r=0,854
$$




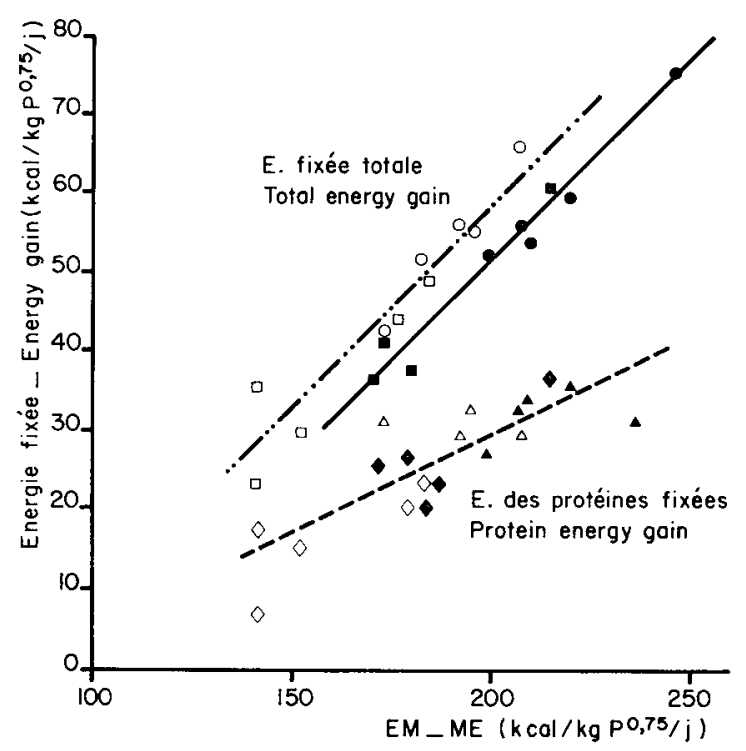

FIG. 3. - Variations des quantités d'énergie fxée totale et sous forme de protéines par les veaux à l'age de ro semaines, en fonction de la quantité d'EM ingérée.

Variations with $M E$ intake in total energy gain and protein energy gain of the ro week-old calves.

Veaux allaités nourris ad libitum $(\bullet \Delta)$ ou restreints $(\bigcirc \Delta)($ Milk-fed calves fed ad libitum $(\bullet \Delta)$ or 75 p. roo of ad lib (OA).

Veaux sevrés précocement nourris ad libitum ( $\mid \downarrow$ ) ou restreints ( $(\diamond)$ ) (Early weaned calves fed ad libitum ( $\diamond)$ or 75 p. Ioo of ad lib (口 $\diamond)$.

Energie fixée totale par les veaux nourris ad libitum (Y) ou restreints ( $Y^{\prime}$ ) (Total energy gain of the ad libitum (Y) or 75 p. Ioo of ad lib (Y') fed calves).

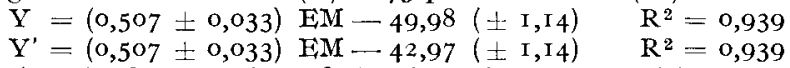

Energie des protéines fixées (Protein energy gain)

$\mathrm{Y}=(0,249 \mathrm{I} \pm 0,040) \mathrm{EM}-20,37( \pm 4,42) \quad \mathrm{r}=0,823$

\section{TABLEAU 4}

Variations du vendement $(\mathrm{kg})$ d'utilisation de l'EM pour la croissance et $d u$ besoin d'entretien des veaux en fonction de l'âge et du niveau d'alimentation

Variations with age and feeding level in $M E$ efficiency for growth ( $k g$ ) and maintenance requirement of calves

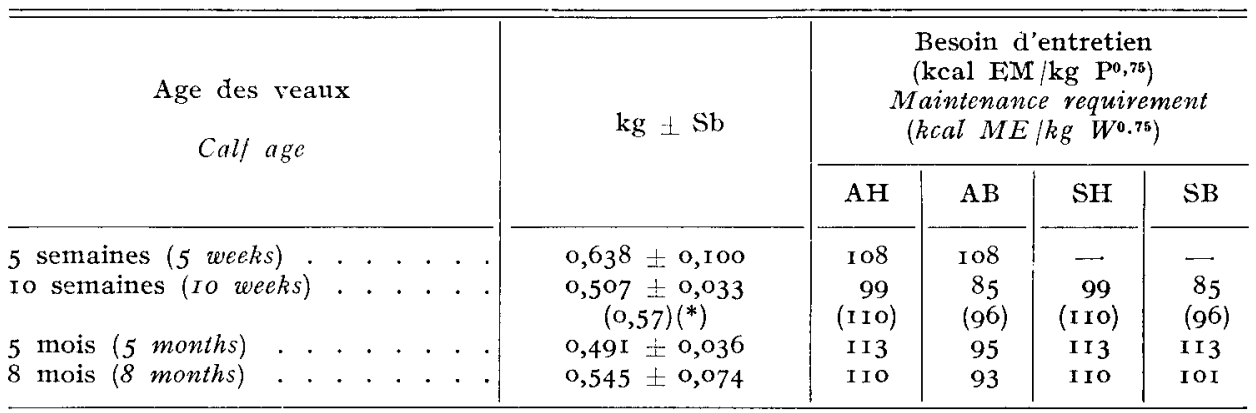

(*) Valeur calculée en prenant IIo et $96 \mathrm{kcal} \mathbf{E} \mathbf{M} / \mathrm{kg} \mathbf{P}^{\mathbf{0 . 7 5}}$ comme besoin d'entretien (Calculated value using $I I O$ and 96 kcal $M E / k g W^{0.75}$ as maintenance requirement). 


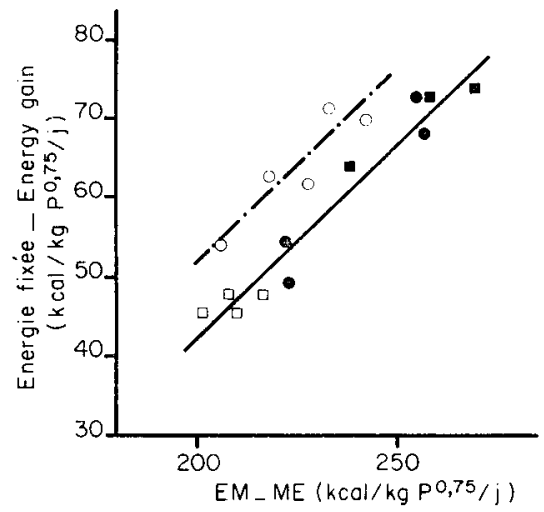

FIG. 4. - Variations des quantités d'énevgie fixée par les veaux à l'age de 5 mois, en fonction de la quantité d'EM ingérée.

Variations with $M E$ intake in total energy gain of the 5 month-old calves.

Veaux allaités nourris ad libitum (AH : - ) ou restreints (AB : O) (Milk-fed calves ad libitum $(A H: \bullet)$ or 75 p. Ioo of ad lib $(A B: 0)$.

Veaux sevrés précocement nourris ad libitum (SH : 回) ou restreints (SB : $\square$ ) (Early weaned calves fed ad libitum ( $S H:$ 回) or 75 p. Ioo of ad lib (SB: $\square$ ).
$\mathrm{AH}+\mathrm{SH}+\mathrm{SB}$
E. fixée $=(0,491 \pm 0,036) \mathrm{EM}-55,52( \pm 0,87)$
E. fixée $-(0,49 \mathrm{I} \pm 0,036)$ EMI $-46,60( \pm 1,30)$
$\mathrm{R}^{2}=0,93$
$\mathrm{AB}$

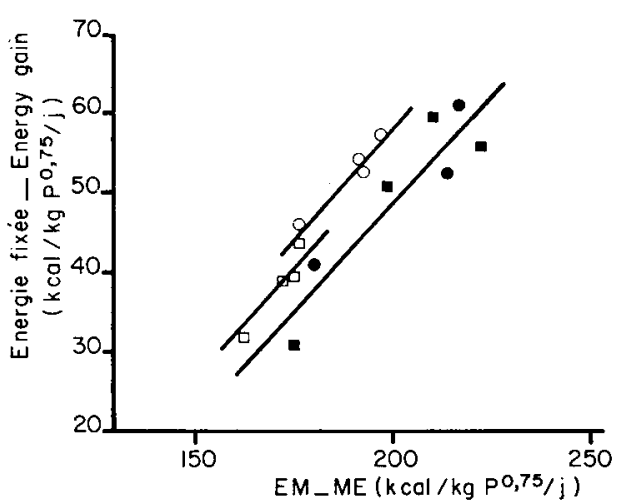

FIG. 5. - Variations des quantités d'énergie fixée par les veaux à l'âge de 8 mois, en fonction de la quantité d'EM ingérée.

Variations with ME intake in total energy gain of the 8 month-old calves.

Veaux allaités, nourris ad libitum (AH : -) ou restreints (AB : O) (Milk-fed calves fed ad libitum $(A H: \bullet)$ or $75 p$. roo of ad lib $(A B: O)$.

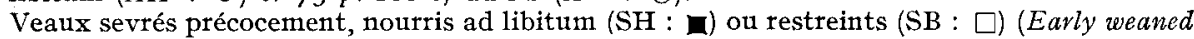

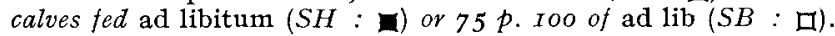
$\mathrm{AH}+\mathrm{SH}: \mathrm{E}$. fixée $=(0,545 \pm 0,074) \mathrm{EM}-59,87( \pm \mathrm{I}, 59)$
$\mathrm{AB} \quad: \mathrm{E}$. fixée $=(0,545 \pm 0,074) \mathrm{EM}-50,65( \pm \mathrm{I}, 78)$
$\mathrm{SB} \quad: \mathrm{E}$. fixée $=(0,545 \pm 0,074) \mathrm{EM}-54,99( \pm 2,26)$
$\mathbf{R}^{2}=0,83 \mathbf{I}$
$\mathbf{R}^{2}=0,83 \mathbf{I}$
$\mathbf{R}^{2}=0,83 \mathbf{I}$

A l'âge de 8 mois, les rations sont les mêmes qu'à l'âge de 5 mois. L'analyse de covariance permet de distinguer 3 populations différentes (fig. 5). Les rendements d'utilisation de 1'EM ne sont pas significativement différents entre les lots $\mathrm{AH}$ et $\mathrm{SH}$, d'une part, et le lot $\mathrm{AB}$ d'autre part. La pente commune des régressions conduit à un rendement $(\mathrm{kg})$ de $0,545 \pm 0,074$, plus élevé qu'à l'âge de 5 mois. 
Les mioyennes ajustées sont significativement différentes pour les lots $\mathrm{AH}$ et $\mathrm{SH}$ d'une part et $A B$ d'autre part, mais non significativement différentes $\left(P<0, I_{5}\right)$ pour le lot SB par rapport aux autres lots; elles se traduisent par des différences de besoin d'entretien.

\section{Besoin d'entretien des veaux}

Le besoin d'entretien est, par définition, la quantité d'EM, calculée par la régression de l'énergie fixée en fonction de 1'EM, qui correspond à une énergie fixée nulle. Il n'est pas significativement différent chez les veaux " allaités " et chez les veaux sevrés précocement qui reçoivent l'aliment solide ad libitum, quel que soit leur âge. De plus, il ne diminue pas significativement entre les âges de 5 semaines et 8 mois et s'élève en moyenne à Iro $\mathrm{kcal} / \mathrm{kg} \mathrm{P}^{0,75}$ chez les veaux recevant l'aliment solide ad libitum (tabl. 4).

Cependant, une restriction alimentaire de $25 \mathrm{p}$. roo appliquée pendant une longue période entraîne en moyenne une réduction de I $5 \mathrm{p}$. Ioo du besoin d'entretien des veaux " allaités " aux âges de ro semaines, 5 mois et 8 mois et des veaux sevrés précocement, à l'âge de ro semaines. Fn revanche, la diminution du besoin d'entretien ( 8 p. roo) n'est pas significative chez les veaux sevrés précocement, à 1'âge de 8 mois et elle est nulle à 1'âge de 5 mois.

Variations de l'utilisation de l'EM en fonction de la composition de la ration, du niveau d'alimentation et de l'âge des veaux

Quel que soit 1'âge considéré, les droites de régression de l'énergie fixée en fonction de 1'EM ingérée par les veaux "allaités " ou sevrés précocement et recevant l'aliment solide ad libitum, ne sont pas significativement différentes. Donc le

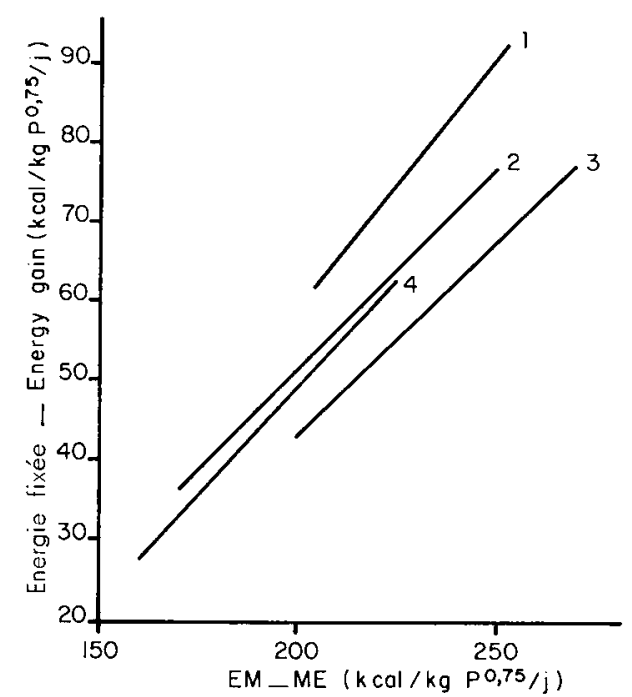

FIG. 6. - Variations des quantités d'EM ingéréc et d'énergie fixée par kg $P^{0,75}$ et par jour, au cous des mesures en chambres respiratoires, par les veaux "allaités" ou sevrés précocement nourris ad libitum, en fonction de l'âge.

Variations with age in $M E$ intake and energy gain (hcal/kg $\left.W^{0,75} / d\right)$ of the milk-fed or early weaned calves fed ad libitum.

(I) 5 semaines; (2) ro semaines; (3) 5 mois; (4) 8 mois

Age $=$ (I) 5 weeks; (2) ro weeks; (3) 5 months; (4) 8 months. 
remplacement de I 5 à I 8 p. Ioo de l'énergie brute, soit de 20 à 25 p. roo de 1'EM, de l'aliment solide par celle de 1'aliment d'allaitement ne modifie pas significativement l'utilisation de 1'EM de la ration quand elle est distribuée ad libitum.

Par ailleurs, le rendement $(\mathrm{kg})$ d'utilisation de 1'EM n'est pas significativement différent chez les veaux recevant l'aliment solide ad libitum ou en quantité limitée, quel que soit leur âge. Cependant, compte tenu des différences de besoin d'entretien, pour une même quantité d'EM ingérée par $\mathrm{kg} \mathrm{P}^{0,75}$, les veaux " allaités " restreints fixent davantage d'énergie que les veaux nourris ad libitum : + I5 + I 5 et 21 p. Ioo aux âges de Io semaines, 5 mois et 8 mois respectivement $(\mathrm{P} \leqslant 0, \mathrm{O} I)$. En revanche, chez les veaux sevrés précocement, l'augmentation de I 5 p. I 00 observée à l'âge de Io semaines n'est que de 8 p. 100 à l'âge de 8 mois $(\mathrm{P} \leqslant \mathrm{O}, \mathrm{I} 5)$ et est nulle à l'âge de 5 mois.

Enfin, la quantité d'énergie fixée pour une même quantité d'EM ingérée par $\mathrm{kg} \mathrm{P}^{0,75}$, varie avec l'âge des veaux. Chez les animaux recevant l'aliment solide ad libitum, et pour l'ingestion moyenne d'EM des différents groupes, les quantités d'énergie fixée (fig. 6) sont comparables aux âges de ro semaines et de 8 mois (respectivement 59,5 et $57,8 \mathrm{kcal} / \mathrm{kg} \mathrm{p}^{0,75}$ ) $\mathrm{m}_{1}$ ais plus élevées à 1'âge de 5 semaines $(68,8)$ et plus faible à l'âge de 5 mois $\left(5^{\circ}, 7\right)$. Les variations sont analogues chez les veaux recevant l'aliment solide en quantité limitée (fig. 7).

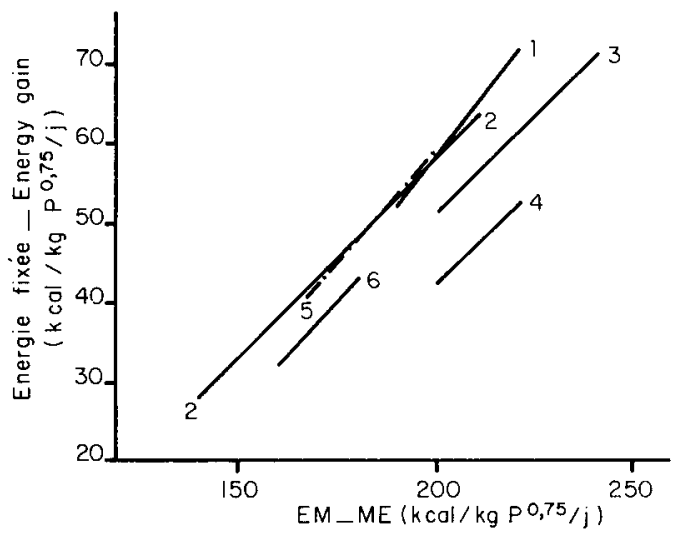

FIG. 7. - Variations des quantités d'EM ingérée et d'énergie fixée par $k g P^{0,75}$ et par jour, au cours des mesures en chambres respiratoires, par les veaux "allaités ou sevrés précocement et restreints, en fonction de l'age.

Variations with age in $M E$ intake and cnergy gain (kcal/kg $\left.W^{0.75} / d\right)$ of the milk-fed $(A B)$ or early weaned $(S B)$ calves ted 75 p. Ioo of ad libitum.

(I) 5 semaines; (2) Io semaines; (3) AB : 5 mois;

(4) $\mathrm{SB}: 5$ mois ;(5) AB : 8 mois; (6) $\mathrm{SB}: 8$ mois.

Age : (I) weeks; (2) Io weeks; (3) Al3: 5 months;

(4) $S B: 5$ months; (5) $A B: 8$ months; (6) $S B: 8$ months.

Quantités de protéines fixées

Les bilans azotés sont satisfaisants chez les veaux âgés de 5 ou ro semaines; ils conduisent à des quantités de protéines fixées cohérentes avec le gain de poids vif (en moyenne I $94 \mathrm{~g} / \mathrm{kg}$ ) et supérieures de ro p. roo environ aux valeurs obtenues par les méthodes des abattages chez des veaux préruminants de poids comparables (NEESE et KIRSCHGESSNER, I 975). Fn revanche, aux âges de 5 et 8 mois, les quan- 
tités de protéines fixées par les veaux sont très variałles pour un même lot et pour des quantités d'EM ingérée voisines; parfois, elles ne sont pas en rapport avec le gain de poids des animaux. Cette variabilité traduit probablement des erreurs dans les bilans azotés malgré les précautions prises; aussi, les bilans azotés obtenus aux âges de 5 et 8 mois n'ont-ils pas été pris en considération.

A l'âge de 5 semaines, la quantité d'énergie fixée sous forme de protéines augmente linéairement avec la quantité d'EM ingérée, mais moins rapidement que la quantité d'énergie totale (fig. 2). Par suite, la proportion d'énergie sous forme de protéines diminue de 65 à $5 \mathrm{I}$ p. roo lorsque les quantités d'énergie ingérées augmentent et que le gain de poids vif passe de 700 à $\mathrm{I}$ ooo g par jour environ. Le phénomène est comparable à l'âge de ro semaines (fig. 3) et il n'y a pas de différence significative entre les veaux " allaités et les veaux sevrés précocement. L'énergie des protéines représente en moyenne $57 \mathrm{p}$. Ioo de l'énergie fixée totale chez les veaux nourris ad libitum et $53 \mathrm{p}$. roo chez les veaux restreints (différence non significative).

\section{Utilisation de l'énergie métabolisable par les moutons}

Pour chaque niveau d'alimentation, les quantités d'énergie fixées par les 3 groupes de 2 moutons sont assez différentes mais se classent dans le même ordre (fig. 8). On a donc calculé le rendement $(k f)$ d'utilisation de 1'F,M pour l'engraissement en faisant le rapport entre la différence d'énergie fixée et la différence d'EM ingérée entre les 2 niveaux d'alimentation.

Les valeurs de $k f$ sont très voisines pour les 3 groupes de 2 moutons $(0,5$ Io $+0,008)$. Ce rendement est très proche de la valeur $(0,494)$ que l'on peut calculer à l'aide de la relation générale proposée par BLAXTER (1974). L'énergie des protéines fixées représente en moyenne $26,5 \pm 5,0 \mathrm{p}$. Ioo de l'énergie fixée totale au haut niveau d'alimentation. Le besoin d'entretien de ces moutons est en moyenne de $95,0 \pm 7,2 \mathrm{kcal}$ d'EMI par $\mathrm{kg}$ de poids métabolique $\left(\mathrm{P}^{0,75}\right)$. Cette valeur est égale à celles obtenues par GRAHAm et SEARLE (I972) pour des moutons âgés de I5 mois et par Vermorei, Bouvier et DeMARQUiliy (I974) pour des moutons âgés de 2 ans. En revanche, elle est plus élevée que la valeur $(76 \mathrm{kcal} \mathrm{d}$ 'EM $/ \mathrm{kg}$ $\mathrm{P}^{0,75}$ ) proposée par l'A.R.C. (I965) pour des moutons âgés de 2 ans.

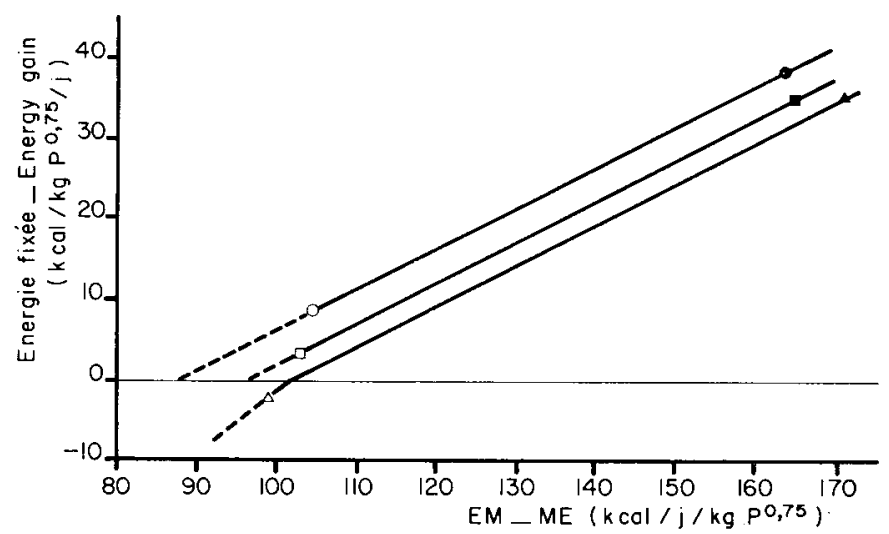

FIG. 8. - Variations des quantités d'énorgie fixéc par les 3 groupes de 2 moutons adultes en fonction des quantités d'éncrgie métabolisable ingérée.

Variation in energy gain with $M E$ intake in the 3 pairs of sheep used. 


\section{Discussion}

\section{Utilisation digestive de l'aliment solide}

Les digestibilités de l'énergie et de l'azote de l'aliment solide ne sont pas modifiées par l'ingestion de lait et on retrouve pour ce dernier les valeurs obtenues chez le veau préruminant. Ce résultat est en accord avec les conclusions de PrESTON, ARChibal, et Trnkler (I967). De plus, la production de méthane, rapportée à l'aliment solide est la même chez les veaux "allaités " et chez les veaux sevrés précocement. Il n'existe donc pas de phénomène de digestibilité associative entre le lait et l'aliment solide. Cette conclusion s'explique bien si on admet que le lait continue d'emprunter la gouttière cesophagienne et n'est pas soumis aux fermentations microbiennes dans le rumen, mais elle suppose que les phénomènes de salivation, de rumination, de motricité qui interviennent dans la digestion microbienne de l'aliment solide, sont comparables chez les veaux " allaités " et chez les veaux sevrés précocement.

L,es digestibilités de l'énergie et de l'azote de l'aliment solide ne varient pas significativement non plus avec l'âge des veaux et sont comparables aux valeurs obtenues chez les moutons adultes pour le même niveau d'alimentation (tabl. 5). Ces résultats sont en accord avec ceux de PRESTON, Archibald et TINKLER (r957), Blaxter, Clapperton et Wainman (r966), Mc Culiough (I970), Johnson, et EILIOTT (I973), mais en contradiction avec ceux de JENTSCH, WITTENBURG et SChiEmann (I976) qui ont obtenu pour 44 rations des CUD de l'énergie et de l'azote inférieurs de 4,6 et 9,6 points respectivement chez des taurillons par rapport aux moutons adultes. Il est possible que ces différences dépendent à la fois de la composition de la ration (nature du fourrage et proportion d'aliment concentré) et des écarts de niveau d'alimentation (VERMOREL, et BICKEL, I980).

Lorsque le niveau d'alimentation augmente d'une unité, les CUD de l'énergie et de l'azote diminuent de 2,7 et 3,0 points respectivement. Ces variations sont égales à celle ( 3,0 points) obtenue par Schiemann, Jentsch et WitTtenburg (I97I) chez des vaches laitières, mais inférieures à celle obtenue par TYRRELL, MOE et OLTJEN (I974) chez des génisses ( 5,7 points), alors que BLAXTER, ClapPERTON et WAINMAN (I 966) n'avaient pas observé de variation significative chez des bouvillons.

Les pertes d'énergie sous forme de méthane rapportées à l'énergie de l'aliment solide ingéré augmentent avec 1'âge des animaux (tabl. 5). Cette évolution peut correspondre à une augmentation de la digestion de l'aliment solide dans le rumen au détriment de la digestion intestinale, en relation avec le développement allométrique positif des réservoirs digestifs; elle expliquerait la variation des proportions d'acides gras volatils dans le jus de rumen observée par SCHIEMANN et al. (I976). En conséquence, le rapport EM/ED diminue lorsque l'âge des veaux avance (tabl. 5), comme l'a également observé Demchenko (I969).

La concentration en $\operatorname{EM}(q)$ de l'aliment solide diminue de I,5 point entre les âges de ro semaines et 8 mois, mais elle est en moyenne comparable à la valeur obtenue chez les moutons adultes, pour le même niveau d'alimentation (tabl. 5) ce qui confirme les données de SchiEMANN et al. (I976). Elle diminue également de $I, 9$ point lorsque le niveau d'alimentation augmente d'une unité. La diminution de la digestibilité due à l'augmentation du niveau d'alimentation est donc partiel- 
TABLEAU 5

Variations de la digestibilité et de la concentration en énergie métabolisable de l'aliment solide chez les veaux en croissance, en fonction de l'âge; comparaison aux valeurs obtenues chez les moutons adultes au haut niveau d'alimentation.

Variations with age in energy and nitrogen digestibility and energy metabolizability of the solid feed in growing calves. Comparison with adult sheep fed at the high feeding level.

\begin{tabular}{|c|c|c|c|c|c|}
\hline Animaux & \multicolumn{4}{|c|}{ Veaux - Calves } & \multirow{2}{*}{$\begin{array}{l}\text { Moutons } \\
\text { Sheep }\end{array}$} \\
\hline $\mathrm{AGE}-A g e$ & $\begin{array}{c}\text { Io se- } \\
\text { maines } \\
\text { to weeks }\end{array}$ & $\begin{array}{l}5 \text { mois } \\
5 \text { months }\end{array}$ & $\begin{array}{l}8 \text { mois } \\
8 \text { months }\end{array}$ & $S x y\left({ }^{*}\right)$ & \\
\hline $\begin{array}{l}\text { CUD Energie (E. digestibility) } \\
\text { CUD Azote (N.digestibility) } \\
\text { ECH } \% \text { E. ingérée }\left(\mathrm{CH}_{4} E \% \text { IE) }\right. \\
\mathrm{IM} \% \mathrm{~F} \text {. ingérée }(M E \% I E) \\
\mathrm{IM} / \mathrm{ED}(M E / D E) .\end{array}$ & $\begin{array}{l}70,4 \\
71,8 \\
3,76 \\
63,8 \\
0,906\end{array}$ & $\begin{array}{l}70,9 \\
73,3 \\
4,16 \\
63,3 \\
0,892\end{array}$ & $\begin{array}{l}71,2 \\
71,3 \\
5,22 \\
62,2 \\
0,874\end{array}$ & $\begin{array}{l}2,0 \\
2,0 \\
0,75 \\
2,0 \\
--\end{array}$ & $\begin{array}{l}72,2: 1,0 \\
72,2 \geq 0,6 \\
6,02 \pm 1,00 \\
62,5 \pm 1,2 \\
0,865\end{array}$ \\
\hline
\end{tabular}

Ces valcurs ont été calculćes en estimant à 93 les CUD de l'énergie et de l'azote de l'aliment d'allaitement (cf). texte). Élles ont été corrigées pour être ramenées au niveau d'alimentation des moutons adulte$\left(63, \mathrm{I} \mathrm{g}\right.$ de MS d'aliment solide par $\left.\mathrm{kg} \mathbf{P}^{\mathbf{0}, 75}\right)$ à l'aide des coefficients obtenus pour NI dans les relations is 2 et 4 (These values were calculated using 9.3 as energy and nitrogen digestibility coefficient of the milk substitute (VERmores, Bouvier and GEAY, 1976). They were corrected for the feeding level of the sheep (6.3.I $\mathrm{gl} / \mathrm{M} / \mathrm{kg}$ $\left.W^{075}\right)$ sing the coefficients of $N I$ found in the velationships 1,2 and $\left.f\right)$.

(*) Sxy : ćcart-type résiduel des régressions $1,2,3$ et 4 (Sxy: residual standard deviations of the rolationships $1,2,3$ and 4 ).

lement compensée, mais de façon moins importante que ce qu'ont trouvé ScHıEMANN, JENTSCH et WITTENBURG (I97I) sur vaches laitières. Ces résultats confirment 1'idée que les teneurs en EM des aliments déterminées sur moutons adultes sont utilisables pour les ruminants en croissance, tout du moins pour des niveaux d'alimentation comparables.

\section{Utilisation de l'EM pour la croissance et efficacité alimentaire}

Les observations ont été effectuées sur un nombre réduit d'animaux pour chaque traitement en raison de la difficulté et de la durée des mesures de bilans énergétiques, ce qui conduit à des variabilités assez élevées du rendement d'ulitisation de l'EM et de l'efficacité alimentaire. De plus, la proportion de l'énergie ingérée apportée par l'aliment d'allaitement diminue lorsque l'âge des veaux augmente, ce qui ne permet pas de distinguer facilement l'effet dû à l'âge. Cependant, la répétition des mesures des bilans à plusieurs stades de croissance permet de tirer quelques conclusions sur l'effet des traitements étudiés. 


\section{- Influence de l'apport de lait}

Chez les veaux recevant l'aliment solide ad libitum, l'apport de lait n'a eu aucun effet significatif sur la quantité d'énergie fixée (pour une même quantité d'EMI ingérée par $\mathrm{kg} \mathrm{P}^{0,75}$ ), sur le besoin d'entretien et sur le rendement d'utilisation de l'EM pour la croissance, quel que soit l'âge.

La contradiction avec les données de BLACK et TRIBE (I973) sur agneaux peut provenir du fait que l'aliment d'allaitement représente ici une part beaucoup plus faible de la ration. En effet, la différence d'énergie apportée par l'aliment d'allaitement entre les 2 traitements n'est en moyenne que de 16 p. roo de l'énergie brute ingérée, soit $22 \mathrm{p}$. Ioo de l'EM. Cependant, si on considère que le rendement d'utilisation de 1'EM de l'aliment d'allaitement est voisin de $0,68 \mathrm{che} z$ le Veau préruminant et que celui de l'aliment solide est de $0,5 \mathrm{I}$ chez le mouton adulte, cet apport de lait devrait théoriquement entraîner une amélioration de 0,04 point $(0,68-0,5 \mathrm{I}) \times 0,22) \mathrm{du}$ rendement de 1 'EM et une augmentation d'environ 8 p. roo de la quantité d'énergie fixée.

Les résultats des bilans sont confirmés par les performances zootechniques des veaux. L'apport de lait n'a entraîné une amélioration de l'efficacité d'utilisation de 1'EM que chez les animaux restreints et surtout au cours des premiers mois. Ce phénomène peut résulter $\mathrm{du}$ fait que les veaux ont reçu une ration riche en concentrés et que les animaux " allaités " recevant l'aliment d'allaitement ad libitum ont ingéré des quantités importantes d'EM et d'acides gras longs, ce qui a pu provoquer un engraissement excessif. Cette hypothèse rejoint les résultats des travaux de LE NEINDRE et al. (à paraître) montrant que l'amélioration apportée par le lait est moins importante avec des rations comportant des aliments concentrés qu'avec de l'herbe seule.

\section{- Influence de la restriction alimentaire}

Chez tous les veaux "allaités " et à l'âge de Io semaines chez les veaux sevrés précocement, la restriction alimentaire ( $77 \mathrm{p}$. Ioo de 1'E,M ingérée par $\mathrm{kg} \mathrm{P}^{\mathbf{0}, 75}$ ) n'a pas entraîné de modification du rendement de 1'EM pour la croissance, mais une réduction de I5 p. IOo en moyenne du besoin d'entretien ( $\left./ \mathrm{kg} \mathrm{P}^{\mathbf{0}, 75}\right)$, en accord avec les résultats de THORBEK et HENCKEL (I976), et une augmentation de l'efficacité alimentaire. Cette dernière peut s'expliquer également par une réduction du dépôt de lipides plus iniportante que celle des protéines (GEAY et RoBEi IN, I979).

En revanche, chez les veaux sevrés précocement qui ingèrent moins d'EM que les veaux "allaités" (- I 8 p. Ioo par jour et - II p. Ioo $/ \mathrm{kg} \mathrm{P}^{0,75}$ ), la restriction alimentaire peut provoquer une réduction plus importante de la fixation de protéines, done du gain de poids, ce qui pourrait expliquer l'absence d'effet sur l'efficacité alimentaire globale notée d'après les performances zootechniques.

\section{- Variation avec l'âge des veaux}

Lorsque l'âge des veaux avance, on observe une réduction importante de l'efficacité alimentaire globale. Celle-ci est due à l'accroissement de la part des dépenses d'entretien. Mais elle résulte aussi, d'une part, de la réduction de la proportion de l'énergie ingérée apportée par l'aliment d'allaitement et, d'autre part, de l'augmentation de la quantité d'énergie fixée par $\mathrm{kg}$ de gain de poids vif, comme le traduit la diminution du rapport "gain de poids vif / $\mathrm{E} M$ de production $"($ tabl. 2). 
I e rendement $(\mathrm{kg})$ d'utilisation de l'EM pour la croissance passe de 0,638 à $0,49 \mathrm{I}$ entre les âges de 5 semaines et 5 mois et cette diminution est à relier à la réduction de la proportion d'énergie apportée par l'aliment d'allaitement. En revanche, $\mathrm{kg}$ est plus élevé à l'âge de 8 mois qu'à l'âge de 5 mois, alors que les veaux reçoivent les mêmes rations. Cette supériorité peut résulter d'une augmentation de la lipogénèse, mais les erreurs sur les bilans azotés ne nous permettent pas de déterminer les quantités de protéines et de lipides fixés. Pour les âges de 5 et 8 mois, le rendement $\mathrm{kg}$ est en moyenne comparable à la valeur $\mathrm{kf}$ obtenue avec les moutons adultes.

Enfin, le besoin d'entretien ne varie pas significativement avec l'âge des veaux. La valeur moyenne (I I kcal d'EM par $\mathrm{kg} \mathrm{P}^{0.75}$ ) est égale à celle trouvée par VERMOREL, Bouvier et Geay (I976), Hoffmann, Jentsch et Schiemann (I977) chez des veaux et des taurillons de race frisonne également.

\section{Conclusion}

Cette étude a permis d'apporter un certain nombre d'informations sur l'utilisation digestive et métabolique des rations par le Veau en croissance. La digestibilité de l'aliment solide utilisé n'est pas modifiée au cours de la croissance et est égale à la valeur obtenue chez des moutons adultes, pour le même niveau d'alimentation; en revanche, les pertes d'énergie sous forme de méthane et dans l'urine augmentent avec 1'âge. Cependant, compte-tenu des faibles variations, les teneurs en EM des aliments déterminées chez des moutons adultes peuvent être utilisées pour les ruminants en croissance. Il n'y a pas d'intéraction entre le lait et l'aliment solide chez le Veau "allaité " au niveau de la digestibilité, mais il serait nécessaire d'étudier les variations de la digestion des constituants de la ration dans las réservoirs gastriques et dans l'intestin du Veau, au cours de la croissance, pour mieux connaitre les variations des produits terminaux de la digestion.

L'apport de lait, représentant environ 22 p. Ioo de 1'EM de la ration, n'a pas entrainé de modification significative de l'utilisation de 1'EM (efficacité alimentaire et rendement de l'E,M pour la croissance), mais il s'agissait d'une ration riche en concentrés et très appétible qui a probablement entraîné un engraissement important des veaux recevant l'aliment solide ad libitum. Il serait intéressant d'étudier l'utilisation digestive et métabolique de ces rations au cours de la période encadrant le sevrage et pendant laquelle les quantités d'aliments ingérées par le Veau sont réduites et les proportions des produits terminaux de la digestion fortement morifiées.

$$
\text { Accepté pour publication en janvier } 1980 .
$$

\section{Summary}

\section{Utilization of feed energy by ruminant calves; effects of milk intake, feeding level and age}

Four groups of 5 male Friesian calves were fed on two feeding levels (ad libitum : $\mathrm{H}$ or 75 p. roo of $a d l i b .:$ B) and weaned either at ten weeks of age (SH and SB groups), or fed 6 or 4.5 I milk in addition to a solid feed ( $A H$ and $A B$ groups) (fig. I and table $I$ ). The same feed composed of $40 \mathrm{p}$. I 100 cobbed hay and $60 \mathrm{p}$. I 00 concentrate was also given to 6 mature sheep on two feeding levels successively ( 1.08 and 1.76 times maintenance). Fnergy balances were determined by indirect calorimetry in sheep and in $5^{-10}$ week-and $5^{-8}$ month-old calves. 
No associative effect was observed between milk and solid feed. Energy and nitrogen digestibility and energy metabolizability of the solid feed were not significantly different in growing calves and adult sheep for the same feeding level, whereas methane and urinary energy (related to energy intake) increased with age (tables 3 and 5).

The overall efficiency of ME (weight gain/ME) (table 2) and the partial efficiency of ME utilization for growth $(\mathrm{kg})$ were not significantly different in the milk-fed calves and in the early weaned ones, at any growth stage. Furthermore $\mathrm{kg}$ was not significantly altered by the reduction of food intake. However, it decreased from 0.638 in the 5 week-old calves to $0.49 \mathrm{I}$ in the 5 monthold calves, as the proportion of gross energy supplied by milk was reduced from 86 to 8 p. 100 ; then it increased to 0.545 in the 8 month-old calves (table 4). Nevertheless, these last two values (kg) were not significantly different from the $\mathrm{kf}$ value $(0.5 \mathrm{r})$ obtained in adult sheep.

The maintenance requirement of the ad libitum fed calves (I o $\mathrm{kcal} / \mathrm{kg} \mathrm{W}^{0.75}$ ) was not altered by milk supply and did not vary significantly between 5 weeks and 8 months of age (table 4). However, a 24 p. I oo reduction of food intake along the experimental period was followed by a 15 to $2 \mathrm{I} \mathrm{p}$. IOO increase in energy gain for the same ME intake ( $/ \mathrm{kg} \mathrm{W}^{0.75}$ ), by a I5 p. roo decrease in maintenance requirement of the milk-fed calves (fig. I to 4 ) and an improvement of the overall efficiency of ME (table 2).

\section{Références bibliographiques}

Agricultural Research Council, 196.5. The Nutrient Requirement of Farm Animals, (A.R.C.), 2, Ruminants (Agricultural Research Council, London).

BLACK J. L., TribE D. E., I973. Comparison of ruminal and abomasal administration of feed on the growth and body composition of lambs. Aust. J. Agric. Res., 24, 763-773.

BLAXTER K. L., 1974. Metabolizable energy and feeding systems for ruminants. In H. SwAN and D. LEWIS (Ed.), Proc. Nutr. Conf. Feed Manuf, Nottingham. Butterworths, London, 3-25.

BLAXTER K. L., Clapperton J. L., WAINMAN F. W., i966. Utilization of the energy and protein of the same diet by cattle of different ages. J. Agric. Sci. Camb., 67, 67-75.

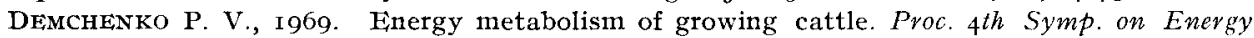
Metabolism of Farm Animals, 2I 3-2I9. K. L. BLAX'TER, J. KIELANOWSKI and GRETA THORHEK (Ed.), Oriel Press Ltd, Newcastle upon Tyne, England.

Es A. J. H. Van, 1970. Simulation of the growth of veal calves fed liquid milk replacers. In: Proc. 5th Symp. Energy Metabolism of Farm Animals, 97-10o. A. Schurch and C. WENk (Ed.), Juris Druck + Verlag, Zürich.

GEAY Y., I97o. Adaptation du mode d'élevage et d'alimentation au type génétique. In "Journées d'étude sur la production de viande par les jeunes bovins ", 95-10o, Éditions S.E.I., C.N.R.A.route de Saint-Cyr, Versailles.

GEAY Y., ROBEIIN J., I979. Variation of meat production capacity in cattle due to genotype and level of feeding: genotype-nutrition interaction. Liv. Prod. Sci, 6, 263-276.

GRAHAM N. Mc, SEARLE T. W., 1972. Balances of energy and matter in growing sheep at several ages, body weight and planes of nutrition. Aust. J. Agric. Res., 23, 97-108.

Guilhermet R., Patureau-Mirand P., Toulize R., Paruel.le J. L., I977. Utilisation de la gouttière cesophagienne pour éviter la dégradation dans le rumen, de mélanges de lactose et de caséine, chez le veau ruminant. Ann. Biol. anim., Bioch., Biophys., 17, 543-547.

Horimann L., Jentsch W., Schiemann R., i977. Die Verwertung der Futterenergie durch wachsende Bullen. Arch. Tievernähr., 27, 545-562.

Jenstch W., WitTendurg H., Schiemann R., I976. Die Verwertung der Futterenergie durch wachsende Bullen. Arch. Tierernähr., 26, 575-585.

Johnson P. T. C., ELLIOTT R. C., 1973. Dietary energy intake and utilization by young Friesland calves. Rhod. J. agric. Res., 11, II-33

LE Neindre P., PETIT M., Mulrer A., I976. Quantités d'herbe et de lait consommées par des veaux au pis. Ann. Zootech., 25, 521-53r.

Mc Cullough T. A., I970. A study of the effect of supplementing a concentrate diet with roughages of different quality on the performance of Fricsian steers. I. Voluntary food intake and food utilization. J. Agric. Sci. Camb., 75, 327-345.

NFESSE K. R., KIRChGESSNER M., I975. Experimentelle Untersuchungen zur Nährstoffetention des Kalbes in Verlauf der Mast. Z. Tierphysiol., Tievernähr., Futtermittelk., 36, 23I-240.

Preston T. R., ARchibald J. D. H., TINkLER W., I957. The digestibility of grass by young calves. J. A gric. Sci., 48, 259-265. 
Schiemann R., JentSch W., WiTTENBURG H., I97I. Züi Abhängigkeit der Verdaulichkeit der Ennergie und der Nährstoffe von der Hôhe der Futterraufnahme und der Rationszusammensetzung bei Milchkühen. Arch. Tievernähr., 21, 223-240.

Schiemani R., Jentsch W., Wittendurg H., HoffmanN L., I976. Die Verwertung der Futterenergie durch wachsende Bullen. I. Versuchsanlage und Ergebnisse des Energie-und Stoffumsatzes. Arch. Tierernähr., 26, 7, 49I-5I 7.

ThorbeK G., HENCKE, S., r976. Studies on energy requirement for maintenance in farm

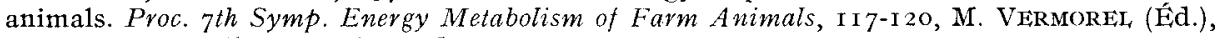
G. DF BUSSAC, Clermont-Ferrand.

TYRRELI H. F., MOE P. W., OLTJEN R. R., I974. Energetics of growth and fattening compared to lactation in cattle. Proc. 6th Symp. on Energy Metabolism of Farm Animals, 205-208, K. H. MENkE, H. J. LANTZSCH and J. R. REICHL (Ed.), Universität Hohenheim, Dokumentationsstelle.

VERMOREI, M., BICKEL, H., 1980. Utilization of feed energy by growing ruminants. Ann. Zootech. (numéro spécial, sous presse).

Vermorel M., Bouvier J. C., Bonnet Y., Fauconneau G., i973. Construction et fonctionnement de 2 chambres respiratoires du type "circuit ouvert" pour jeunes bovins. Ann. Biol. anim., Bioch., Biophys., 13, 659-681.

Vérmoreit M., Bouvier J. C., Demarquiliy C., i974. Influence du mode de conditionnement des fourrages déshydratés sur leur valeur énergétique nette pour le mouton en croissance. Proc. 6th Symp. on Energy Metabolism of Farm Animals, 213-2 I6. K. H. MENkE, H. J. LANTZSCH and J. R. REICHL (Ed.), Universität Hohenheim, Dokumentationsstelle.

VERMOREL, M., BOUVIER J. C., GEAY Y., I976. The effect of genotype (normal or double muscled charolais and friesian) on energy utilization by growing cattle at 2 and 16 months of age. Proc. 7th Symp. on Energy Metabolism of Farm Animals, 2 I7-220. M. VERMOREI, (Ed.), G. DE BUSSAC, Clermont-Ferrand.

Vermorel, M., Bouvier J. C., Thivend P., Toullec R., I974. Utilisation énergétique des aliments d'allaitement par le veau préruminant à l'engrais, à différents poids. Proc. 6th $S y m p$. on Energy Metabolism of Farm Animals, I43-I46. K. H. MENikE, H. J. LANTzSCH and J. R. REICHI, (Ed.), Universität Holnenheim, Dokumentationsstelle. 